\title{
A note on global existence of strong solution to the 3D micropolar equations with a damping term
}

\author{
Wen Wang ${ }^{1}$ and Yunchong Long ${ }^{2 *}$
}

\section{"Correspondence:}

longyunchong@gmail.com

${ }^{2}$ Department of Propaganda,

Changchun University of Chinese

Medicine, Changchun, China

Full list of author information is

available at the end of the article

\section{Springer}

\begin{abstract}
This paper studies the Cauchy problem of the 3D incompressible micropolar equations with a damping term $\sigma|u|^{\beta-1} u(\sigma>0,1 \leq \beta<3)$. It is shown that the strong solutions exist globally for any $1 \leq \beta<3$.
\end{abstract}

Keywords: Micropolar equations; Damping; Global regularity

\section{Introduction}

We consider the Cauchy problem of the 3D incompressible micropolar equations with a nonlinear damping term $\sigma|u|^{\beta-1} u(\sigma>0,1 \leq \beta<3)$ (see [5]):

$$
\left\{\begin{array}{l}
u_{t}+u \cdot \nabla u-(v+\kappa) \Delta u+\sigma|u|^{\beta-1} u+\nabla p=2 \kappa \nabla \times w, \\
w_{t}+u \cdot \nabla w+4 \kappa w-\gamma \Delta w-\mu \nabla \operatorname{div} w=2 \kappa \nabla \times u, \\
\operatorname{div} u=0, \\
u(x, 0)=u_{0}(x), \quad w(x, 0)=w_{0}(x),
\end{array}\right.
$$

where $u \in \mathbb{R}^{3}, w \in \mathbb{R}^{3}, p \in \mathbb{R}$ are the velocity field of fluid, the field of microrotation representing the angular velocity of the rotation of the fluid particles and the scalar pressure, respectively. The parameter $v$ is the kinematic viscosity; $\kappa$ is the microrotation viscosity; $\gamma$ and $\mu$ are the angular viscosities; $\sigma$ is the damping coefficient.

When $w=0$ and $\kappa=0$, the system (1.1) is reduced to the incompressible damped Navier-Stokes equations which was studied firstly by Cai and Jiu [1]. They proved that the corresponding equations admit a global weak solution for any $\beta \geq 1$ and a global strong solutions for $\beta \geq \frac{7}{2}$. Moreover, the uniqueness was shown for any $\frac{7}{2} \leq \beta \leq 5$. We refer to $[3,4,6-8]$ for more results on the Navier-Stokes equations with a damping term.

Recently, the Cauchy problem (1.1) was considered by Ye [5]. It was proved that system (1.1) admits global strong solution for any $\beta \geq 3$. In this paper, we aim to study existence of global solutions under some smallness condition of the initial data for any $1 \leq \beta<3$. Before stating our main results, we firstly state the local strong solutions to (1.1), which can be proved by the similar technique as in [2]. Thus, we omit the details.

(c) The Author(s) 2021. This article is licensed under a Creative Commons Attribution 4.0 International License, which permits use, sharing, adaptation, distribution and reproduction in any medium or format, as long as you give appropriate credit to the original author(s) and the source, provide a link to the Creative Commons licence, and indicate if changes were made. The images or other third party material in this article are included in the article's Creative Commons licence, unless indicated otherwise in a credit line to the material. If material is not included in the article's Creative Commons licence and your intended use is not permitted by statutory regulation or exceeds the permitted use, you will need to obtain permission directly from the copyright holder. To view a copy of this licence, visit http://creativecommons.org/licenses/by/4.0/. 
Theorem 1.1 Suppose that $1 \leq \beta<3,\left(u_{0}, w_{0}\right) \in H^{1}\left(\mathbb{R}^{3}\right)$ with $\operatorname{div} u_{0}=0$. Then there exist a small positive time $T_{0}$ and a unique strong solution $(u, w)$ to the Cauchy problem (1.1) in $\mathbb{R}^{3} \times\left(0, T_{0}\right]$.

Now, our main results read as follows.

Theorem 1.2 Assume that $\left(u_{0}, w_{0}\right) \in H^{1}\left(\mathbb{R}^{3}\right)$ with $\operatorname{div} u_{0}=0 .(u, w)(x, t)$ is the corresponding local strong to (1.1). For $1 \leq \beta<3$, let $T^{*}>0$ be a maximal existence time of the solution. If $T^{*}<\infty$, then

$$
\lim _{T \rightarrow T^{*}}\|u\|_{L^{s}\left(0, T ; L^{r}\right)}=\infty, \quad \text { with } \frac{2}{S}+\frac{3}{r} \leq 1,3<r<\infty .
$$

Theorem 1.3 Suppose $\left(u_{0}, w_{0}\right) \in H^{1}\left(\mathbb{R}^{3}\right)$ with $\operatorname{div} u_{0}=0$. If $1 \leq \beta<3$, then there exists a small positive constant $\varepsilon_{0}$ depending only on $\mu, \gamma, \sigma, \kappa$ and $v$, such that if

$$
\left(\left\|w_{0}\right\|_{L^{2}}^{2}+\left\|u_{0}\right\|_{L^{2}}^{2}\right)\left(\left\|\nabla w_{0}\right\|_{L^{2}}^{2}+\left\|\nabla u_{0}\right\|_{L^{2}}^{2}\right) \leq \varepsilon_{0}
$$

the Cauchy problem (1.1) admits a unique global strong solution, satisfying

$$
\begin{aligned}
& (u, w) \in L^{\infty}\left(0, T ; H^{1}\left(\mathbb{R}^{3}\right)\right) \cap L^{2}\left(0, T ; H^{2}\left(\mathbb{R}^{3}\right)\right), \\
& |u|^{\frac{\beta-1}{2}} \nabla u \in L^{2}\left(0, T ; L^{2}\left(\mathbb{R}^{3}\right)\right), \quad \nabla|u|^{\frac{\beta+1}{2}} \in L^{2}\left(0, T ; L^{2}\left(\mathbb{R}^{3}\right)\right) .
\end{aligned}
$$

Remark 1 When $w=0$ and $\kappa=0$, Theorem 1.1 and 1.2 generalize the previous results for the 3D Navier-Stokes equations with a damping term (see $[7,8])$.

\section{The proof of Theorem 1.2}

This section is devoted to the proof of Theorem 1.2. In what follows, $C$ denotes a generic positive constant depending only on $\mu, \gamma, \sigma, \nu, \kappa$ and $\beta$. Let $(u, w)$ be a strong solution of (1.1) on $\mathbb{R}^{3} \times(0, T)$ described in Theorem 1.1. As aforementioned, we shall prove Theorem 1.2 by contradiction arguments. So, from now on we assume otherwise that

$$
\int_{0}^{T^{*}}\|u\|_{L^{r}}^{S} d t=M_{0}<\infty
$$

with $\frac{2}{s}+\frac{3}{r} \leq 1,3<r<\infty$.

First, multiplying $(1.1)_{1}$ and $(1.1)_{2}$ by $u$ and $w$, respectively, integrating (by parts) the resulting equations over $\mathbb{R}^{3}$, we have

$$
\begin{aligned}
\frac{1}{2} \frac{d}{d t} \int\left(|u|^{2}+|w|^{2}\right) d x+\sigma \int|u|^{\beta+1} d x+(v+\kappa) \int|\nabla u|^{2} d x \\
\quad+\gamma \int|\nabla w|^{2} d x+4 \kappa \int|w|^{2} d x+\mu|\operatorname{div} w|^{2} d x \\
=-\int u \cdot \nabla u \cdot u d x-\int u \cdot \nabla w \cdot w d x+2 \kappa \int(\nabla \times w) \cdot u d x+2 \kappa \int(\nabla \times u) \cdot w d x \\
=2 \kappa \int(\nabla \times w) \cdot u d x+2 \kappa \int(\nabla \times u) \cdot w d x
\end{aligned}
$$




$$
=4 \kappa \int(\nabla \times u) \cdot w d x \leq \kappa\|\nabla u\|_{L^{2}}^{2}+4 \kappa\|w\|_{L^{2}}^{2},
$$

which integrate with respect to $t$,

$$
\|u(t)\|_{L^{2}}^{2}+\|w(t)\|_{L^{2}}^{2}+\int_{0}^{t}\left(2 v\|\nabla u\|_{L^{2}}^{2}+2 \gamma\|\nabla w\|_{L^{2}}^{2}\right) d x \leq\left\|u_{0}\right\|_{L^{2}}^{2}+\left\|w_{0}\right\|_{L^{2}}^{2} .
$$

Next, multiplying $(1.1)_{1}$ by $-\Delta u$ and integrating (by parts) the resulting equations over $\mathbb{R}^{3}$. By Hölder's, Young's and the Gagliardo-Nirenberg inequalities, we have

$$
\begin{aligned}
\frac{1}{2} & \frac{d}{d t} \int|\nabla u|^{2} d x+\sigma \beta \int|u|^{\beta-1}|\nabla u|^{2} d x+(v+\kappa) \int|\Delta u|^{2} d x \\
& =\int u \cdot \nabla u \cdot \Delta u d x-2 \kappa \int(\nabla \times w) \cdot \Delta u d x \\
& \leq \frac{v+\kappa}{4}\|\Delta u\|_{L^{2}}^{2}+\frac{2}{v+\kappa} \int|u|^{2}|\nabla u|^{2} d x+\frac{8 \kappa^{2}}{v+\kappa}\|\nabla w\|_{L^{2}}^{2} \\
& \leq \frac{v+\kappa}{4}\|\Delta u\|_{L^{2}}^{2}+\frac{C}{v+\kappa}\|u\|_{L^{r}}^{2}\|\nabla u\|_{L^{2}}^{2}+\frac{8 \kappa^{2}}{v+\kappa}\|\nabla w\|_{L^{2}}^{2} \\
& \leq \frac{v+\kappa}{4}\|\Delta u\|_{L^{2}}^{2}+\frac{8 \kappa^{2}}{v+\kappa}\|\nabla w\|_{L^{2}}^{2}+\frac{C}{v+\kappa}\|u\|_{L^{r}}^{2}\|\nabla u\|_{L^{2}}^{\frac{2(r-3)}{r}}\|\Delta u\|_{L^{2}}^{\frac{6}{r}} \\
& \leq \frac{v+\kappa}{2}\|\Delta u\|_{L^{2}}^{2}+\frac{8 \kappa^{2}}{v+\kappa}\|\nabla w\|_{L^{2}}^{2}+\frac{C}{(v+\kappa)^{2}}\|u\|_{L^{r}}^{\frac{2 r}{r-3}}\|\nabla u\|_{L^{2}}^{2}
\end{aligned}
$$

with arbitrary $r>3$.

Similarly, multiplying $(1.1)_{2}$ by $-\Delta w$ and integrating (by parts) the resulting equations over $\mathbb{R}^{3}$, we have

$$
\begin{aligned}
& \frac{1}{2} \frac{d}{d t} \int|\nabla w|^{2} d x+\gamma \int|\Delta w|^{2} d x+4 \kappa \int|\nabla w|^{2} d x+\mu|\nabla \operatorname{div} w|^{2} d x \\
& \quad=\int u \cdot \nabla w \cdot \Delta w d x-2 \kappa \int(\nabla \times u) \cdot \Delta w d x \\
& \quad \leq \frac{\gamma}{4}\|\Delta w\|_{L^{2}}^{2}+\frac{2}{\gamma} \int|u|^{2}|\nabla w|^{2} d x+\frac{8 \kappa^{2}}{\gamma}\|\nabla u\|_{L^{2}}^{2} \\
& \quad \leq \frac{\gamma}{2}\|\Delta w\|_{L^{2}}^{2}+\frac{8 \kappa^{2}}{\gamma}\|\nabla u\|_{L^{2}}^{2}+\frac{C}{\gamma^{2}}\|u\|_{L^{r}}^{\frac{2 r}{r-3}}\|\nabla w\|_{L^{2}}^{2} .
\end{aligned}
$$

Adding (2.4) and (2.5), by Gronwall's inequality and (2.3), we have

$$
\|\nabla u\|_{L^{2}}^{2}+\|\nabla w\|_{L^{2}}^{2}+\int_{0}^{t}\left(\|\Delta u\|_{L^{2}}^{2}+\|\Delta w\|_{L^{2}}^{2}\right) \leq C\left(\left\|\nabla u_{0}\right\|_{L^{2}}^{2}+\left\|\nabla w_{0}\right\|_{L^{2}}^{2}\right) .
$$

Therefore, if $u \in L^{s}\left(0, T ; L^{r}\right)$ with $\frac{2}{s}+\frac{3}{r} \leq 1$, we can take $\left.(u, w)\right|_{t=T^{*}}$ as the initial data, then the local strong solutions $(u, w)$ can be extended beyond $T^{*}$. This contradicts the assumption that $T^{*}>0$ is the maximal existence time. The proof of Theorem 1.2 is complete.

\section{The proof of Theorem 1.3}

Throughout this section, we denote

$$
C_{0}:=\left\|u_{0}\right\|_{L^{2}}^{2}+\left\|w_{0}\right\|_{L^{2}}^{2}
$$


Let $(u, w)$ be the strong solution to the problem $(1.1)$ on $\mathbb{R}^{3} \times(0, T)$, then one has the following estimates. First, we infer from (2.4) and (2.5) that

$$
\begin{aligned}
\frac{1}{2} \frac{d}{d t} \int\left(|\nabla u|^{2}+|\nabla w|^{2}\right) d x+\sigma \beta \int|u|^{\beta-1}|\nabla u|^{2} d x+(v+\kappa) \int|\Delta u|^{2} d x \\
\quad+\gamma \int|\Delta w|^{2} d x+4 \kappa \int|\nabla w|^{2} d x+\mu|\nabla \operatorname{div} w|^{2} d x \\
=\int u \cdot \nabla u \cdot \Delta u d x+\int u \cdot \nabla w \cdot \Delta w d x-4 \kappa \int(\nabla \times w) \cdot \Delta u d x \\
\leq 4 \kappa\|\nabla w\|_{L^{2}}^{2}+\left(\kappa+\frac{v}{4}\right)\|\Delta u\|_{L^{2}}^{2}+\frac{\gamma}{2}\|\Delta w\|_{L^{2}}^{2} \\
\quad+\frac{1}{v} \int|u|^{2}|\nabla u|^{2} d x+\frac{1}{2 \gamma} \int|u|^{2}|\nabla w|^{2} d x \\
\leq 4 \kappa\|\nabla w\|_{L^{2}}^{2}+\left(\kappa+\frac{v}{4}\right)\|\Delta u\|_{L^{2}}^{2}+\frac{\gamma}{2}\|\Delta w\|_{L^{2}}^{2}+\frac{1}{v}\|u\|_{L^{\infty}}^{2}\|\nabla u\|_{L^{2}}^{2} \\
\quad+\frac{1}{2 \gamma}\|u\|_{L^{\infty}}^{2}\|\nabla w\|_{L^{2}}^{2} \\
\leq 4 \kappa\|\nabla w\|_{L^{2}}^{2}+\left(\kappa+\frac{v}{4}\right)\|\Delta u\|_{L^{2}}^{2}+\frac{\gamma}{2}\|\Delta w\|_{L^{2}}^{2} \\
\quad+C\|u\|_{L^{6}}\|\Delta u\|_{L^{2}}\left(\frac{1}{v}\|\nabla u\|_{L^{2}}^{2}+\frac{1}{2 \gamma}\|\nabla w\|_{L^{2}}^{2}\right) \\
\leq 4 \kappa\|\nabla w\|_{L^{2}}^{2}+\left(\kappa+\frac{v}{2}\right)\|\Delta u\|_{L^{2}}^{2}+\frac{\gamma}{2}\|\Delta w\|_{L^{2}}^{2}+\frac{C}{v^{3}}\|\nabla u\|_{L^{2}}^{6} \\
\quad+\frac{C}{\gamma^{3}}\|\nabla u\|_{L^{2}}^{2}\|\nabla w\|_{L^{2}}^{4} .
\end{aligned}
$$

Then we obtain after integrating (3.2) with respect to $t$

$$
\begin{aligned}
& \sup _{0 \leq s \leq t}\left(\|\nabla u\|_{L^{2}}^{2}+\|\nabla w\|_{L^{2}}^{2}\right)+\int_{0}^{t}\left(\|\Delta u\|_{L^{2}}^{2}+\|\Delta w\|_{L^{2}}^{2}\right) d s \\
& \quad+\int_{0}^{t}\left(\sigma\left\||u|^{\frac{\beta-1}{2}} \nabla u\right\|_{L^{2}}^{2}+\frac{4 \sigma(\beta-1)}{(\beta+1)^{2}}\left\|\nabla|u|^{\frac{\beta+1}{2}}\right\|_{L^{2}}^{2}\right) d s \\
& \leq\left\|\nabla u_{0}\right\|_{L^{2}}^{2}+\left\|\nabla w_{0}\right\|_{L^{2}}^{2}+C_{1} \int_{0}^{t}\left(\|\nabla u\|_{L^{2}}^{6}+\|\nabla u\|_{L^{2}}^{2}\|\nabla w\|_{L^{2}}^{4}\right) d s \\
& \leq\left\|\nabla u_{0}\right\|_{L^{2}}^{2}+\left\|\nabla w_{0}\right\|_{L^{2}}^{2}+C_{1} \sup _{0 \leq s \leq t}\left(\|\nabla u\|_{L^{2}}^{4}+\|\nabla w\|_{L^{2}}^{4}\right) \int_{0}^{t}\|\nabla u\|_{L^{2}}^{2} d s \\
& \leq\left\|\nabla u_{0}\right\|_{L^{2}}^{2}+\left\|\nabla w_{0}\right\|_{L^{2}}^{2}+C_{1} C_{0} \sup _{0 \leq s \leq t}\left(\|\nabla u\|_{L^{2}}^{4}+\|\nabla w\|_{L^{2}}^{4}\right) .
\end{aligned}
$$

Next, define the function $A(t)$ as follows:

$$
A(t):=\sup _{0 \leq s \leq t}\left(\|\nabla u\|_{L^{2}}^{2}+\|\nabla w\|_{L^{2}}^{2}\right) .
$$


Due to the regularity of $u$ and $w$, one can deduce that $A(t)$ is a continuous function on $[0, T]$. According to (3.3), we have

$$
A(t) \leq\left\|\nabla u_{0}\right\|_{L^{2}}^{2}+\left\|\nabla w_{0}\right\|_{L^{2}}^{2}+C_{1} C_{0} A^{2}(t) .
$$

Now, by (3.5), one can prove that

$$
A(t) \leq 3\left(\left\|\nabla u_{0}\right\|_{L^{2}}^{2}+\left\|\nabla w_{0}\right\|_{L^{2}}^{2}\right)
$$

In fact, we assume that

$$
\left(\left\|u_{0}\right\|_{L^{2}}^{2}+\left\|w_{0}\right\|_{L^{2}}^{2}\right)\left(\left\|\nabla u_{0}\right\|_{L^{2}}^{2}+\left\|\nabla w_{0}\right\|_{L^{2}}^{2}\right) \leq \varepsilon_{0}
$$

and set

$$
T_{*}:=\max \left\{t \in[0, T]: A(t) \leq 3\left(\left\|\nabla u_{0}\right\|_{L^{2}}^{2}+\left\|\nabla w_{0}\right\|_{L^{2}}^{2}\right), \forall s \in[0, t]\right\} .
$$

Then we claim that

$$
T=T_{*} .
$$

Otherwise, we have $T_{*} \in(0, T)$. By the continuity of $A(t)$, it follows from (3.5), (3.7)-(3.8) that

$$
\begin{aligned}
A\left(T_{*}\right) & \leq\left\|\nabla u_{0}\right\|_{L^{2}}^{2}+\left\|\nabla w_{0}\right\|_{L^{2}}^{2}+C_{1} C_{0} A^{2}\left(T_{*}\right) \\
& \leq\left\|\nabla u_{0}\right\|_{L^{2}}^{2}+\left\|\nabla w_{0}\right\|_{L^{2}}^{2}+3 C_{1} C_{0}\left(\left\|\nabla u_{0}\right\|_{L^{2}}^{2}+\left\|\nabla w_{0}\right\|_{L^{2}}^{2}\right) A\left(T_{*}\right) \\
& \leq\left\|\nabla u_{0}\right\|_{L^{2}}^{2}+\left\|\nabla w_{0}\right\|_{L^{2}}^{2}+\frac{1}{2} A\left(T_{*}\right),
\end{aligned}
$$

here, we choose $\varepsilon_{0}=\frac{1}{6 C_{1}}$. Thus, from (3.9) we deduce that

$$
A\left(T_{*}\right) \leq 2\left(\left\|\nabla u_{0}\right\|_{L^{2}}^{2}+\left\|\nabla w_{0}\right\|_{L^{2}}^{2}\right) .
$$

This contradicts (3.8). Hence, by virtue of the argument of continuity and (3.10), we can easily get the desired (3.6).

Finally, we ready to give the proof of Theorem 1.2. In fact, due to Theorem 1.1, there is a unique local strong solution $(u, w)$ to Eqs. (1.1). Let $T^{*}$ be the maximal existence time to the solution. We will show that $T^{*}=\infty$. Otherwise, by contradiction, we take $T^{*}<\infty$, then, by Theorem 1.2, we get, for any $(s, r)$ with $\frac{2}{s}+\frac{3}{r} \leq 1,3<r<\infty$,

$$
\int_{0}^{T^{*}}\|u\|_{L^{r}}^{s} d t=\infty
$$

which together with Sobolev's inequality $\|u\|_{L^{6}} \leq C\|\nabla u\|_{L^{2}}$ leads to

$$
\int_{0}^{T^{*}}\|\nabla u\|_{L^{2}}^{4} d t=\infty
$$


On the other hand, by Hölder's inequality, the Gagliardo-Nirenberg inequality, (2.3) and (3.7), we get

$$
\begin{aligned}
\int_{0}^{T^{*}}\|\nabla u\|_{L^{2}}^{4} d t & \leq \sup _{0 \leq t \leq T^{*}}\|\nabla u\|_{L^{2}}^{2} \int_{0}^{T^{*}}\|\nabla u\|_{L^{2}}^{2} d t \\
& \leq C\left(\left\|u_{0}\right\|_{L^{2}}^{2}+\left\|w_{0}\right\|_{L^{2}}^{2}\right)\left(\left\|\nabla u_{0}\right\|_{L^{2}}^{2}+\left\|\nabla w_{0}\right\|_{L^{2}}^{2}\right) \\
& <+\infty
\end{aligned}
$$

contradicting (3.12). This contradiction shows that $T^{*}=\infty$, and thus we obtain the global strong solution of (1.1). This ends the proof of Theorem 1.3.

\section{Acknowledgements}

The authors would like to thank the anonymous reviewers and the Editor for their constructive comments which helped to improve the quality of the paper.

\section{Funding}

Not applicable.

\section{Abbreviations}

Not applicable.

\section{Availability of data and materials}

Not applicable.

\section{Ethics approval and consent to participate}

Not applicable.

\section{Competing interests}

The authors declare that they have no competing interests.

\section{Consent for publication}

Not applicable.

\section{Authors' contributions}

WW and $Y L$ designed the methodology. WW wrote the draft and derived the theorem. $Y L$ reviewed and revised the paper. All authors read and approved the final manuscript.

\section{Author details}

${ }^{1}$ School of Medical Information, Changchun University of Chinese Medicine, Changchun, China. ${ }^{2}$ Department of Propaganda, Changchun University of Chinese Medicine, Changchun, China.

\section{Publisher's Note}

Springer Nature remains neutral with regard to jurisdictional claims in published maps and institutional affiliations.

Received: 9 December 2020 Accepted: 24 July 2021 Published online: 21 August 2021

\section{References}

1. Cai, X., Jiu, Q.: Weak and strong solutions for the incompressible Navier-Stokes equations with damping. J. Math. Anal. Appl. 343, 799-809 (2008)

2. Chae, M., Kang, K., Lee, J.: Existence of smooth solutions to coupled chemotaxis-fluid equations. Discrete Contin. Dyn. Syst. 33, 2271-2297 (2013)

3. Gala, S., Ragusa, M.A.: A new regularity criterion for the Navier-Stokes equations in terms of the two components of the velocity. Electron. J. Qual. Theory Differ. Equ. 2016, 26 (2016)

4. Gala, S., Ragusa, M.A.: On the regularity criterion for the Navier-Stokes equations in terms of one directional derivative. Asian-Eur. J. Math. 10, Article ID 1750012 (2017)

5. Ye, Z.: Global existence of strong solution to the 3D micropolar equations with a damping term. Appl. Math. Lett. 83, 188-193 (2018)

6. Zhang, Z., Wu, X., Lu, M.: On the uniqueness of strong solution to the incompressible Navier-Stokes equations with damping. J. Math. Anal. Appl. 377, 414-419 (2011)

7. Zhong, X.: Global well-posedness to the incompressible Navier-Stokes equations with damping. Electron. J. Qual. Theory Differ. Equ. 2017, 62 (2017)

8. Zhou, Y.: Regularity and uniqueness for the 3D incompressible Navier-Stokes equations with damping. Appl. Math. Lett. 25, 1822-1825 (2012) 